\title{
Separated boundary value problem for fractional differential equations depending on lower-order derivative
}

\author{
Xiaoyou Liu ${ }^{1,2^{*}}$ and Zhenhai Liu
}

\author{
"Correspondence: \\ liuxiaoyou2002@hotmail.com \\ 'School of Science, Guangxi \\ University for Nationalities, Nanning, \\ Guangxi 530006, P.R. China \\ ${ }^{2}$ School of Mathematical Science \\ and Computing Technology, \\ Central South University, Changsha, \\ Hunan 410075, P.R. China
}

\begin{abstract}
We study a new class of boundary value problems of nonlinear fractional differential equations whose nonlinear term depends on a lower-order fractional derivative with fractional separated boundary conditions. Some existence and uniqueness results are obtained by using standard fixed point theorems. Examples are given to illustrate the results.
\end{abstract}

Keywords: fractional differential equations; fractional separated boundary conditions; fixed point theorems; existence

\section{Introduction}

In this paper, we study the existence and uniqueness of solutions for a class of fractional differential equations whose nonlinear term $f$ depends on the lower-order fractional derivative of the unknown function $x(t)$ with the fractional separated boundary conditions given by

$$
\begin{cases}{ }^{c} D^{\alpha} x(t)=f\left(t, x(t),{ }^{c} D^{\beta} x(t)\right), & t \in[0, T], 1<\alpha \leq 2,0<\beta \leq 1, \\ a_{1} x(0)+b_{1}\left({ }^{c} D^{\gamma} x(0)\right)=c_{1}, & a_{2} x(T)+b_{2}\left({ }^{c} D^{\gamma} x(T)\right)=c_{2}, \quad 0<\gamma<1,\end{cases}
$$

where ${ }^{c} D^{q}$ denotes the Caputo fractional derivative of order $q, f$ is a continuous function on $[0, T] \times \mathbb{R} \times \mathbb{R}$ and $a_{i}, b_{i}, c_{i}, i=1,2$ are real constants with $a_{1} \neq 0$ and $T>0$.

Ahmad and Ntouyas [1] investigated the existence of solutions for a fractional boundary value problem with fractional separated boundary conditions given by

$$
\begin{aligned}
& { }^{c} D^{q} x(t)=f(t, x(t)), \quad t \in[0,1], 1<q \leq 2, \\
& \alpha_{1} x(0)+\beta_{1}\left({ }^{c} D^{p} x(0)\right)=\gamma_{1}, \quad \alpha_{2} x(1)+\beta_{2}\left({ }^{c} D^{p} x(1)\right)=\gamma_{2}, \quad 0<p<1,
\end{aligned}
$$

where ${ }^{c} D^{q}$ denotes the Caputo fractional derivative of order $q, f$ is a given continuous function and $\alpha_{i}, \beta_{i}, \gamma_{i}(i=1,2)$ are real constants, with $\alpha_{1} \neq 0$.

In [2] the same authors considered the following fractional differential inclusion:

$$
{ }^{c} D^{q} x(t) \in F(t, x(t)), \quad t \in[0,1], 1<q \leq 2,
$$

with the boundary condition given by (2). Here $F:[0,1] \times \mathbb{R} \rightarrow 2^{\mathbb{R}}$ is a multivalued map.

\section{Springer}

C) 2013 Liu and Liu; licensee Springer. This is an Open Access article distributed under the terms of the Creative Commons Attribution License (http://creativecommons.org/licenses/by/2.0), which permits unrestricted use, distribution, and reproduction in any medium, provided the original work is properly cited. 
Recently, the subject of fractional differential equations has emerged as an important area of investigation. Indeed, we can find numerous applications of fractional order derivatives in engineering and sciences such as physics, mechanics, chemistry, economics and biology, etc. [3-5]. For some recent developments on the existence results of fractional differential equations, we can refer to, for instance, [6-22] and the references therein.

Fractional differential equations whose nonlinear term $f$ depends on a fractional derivative of the unknown function $x(t)$ have not been studied extensively. In this direction, we can see [23, 24] (fractional anti-periodic boundary value problem) and [25] (anti-periodic boundary value problem) for example.

We remark that when the third variable of the function $f$ in (1) vanishes, the problem (1) reduces to the case considered in [1] by Ahmad and Ntouyas.

\section{Preliminaries}

Definition 2.1 ([26]) The Riemann-Liouville fractional integral of order $q$ for a function $f$ is defined as

$$
I^{q} f(t)=\frac{1}{\Gamma(q)} \int_{0}^{t} \frac{f(s)}{(t-s)^{1-q}} d s, \quad q>0
$$

provided the integral exists.

Definition 2.2 ([26]) For a continuous function $f$, the Caputo derivative of order $q$ is defined as

$$
{ }^{c} D^{q} f(t)=\frac{1}{\Gamma(n-q)} \int_{0}^{t}(t-s)^{n-q-1} f^{(n)}(s) d s, \quad n-1<q<n, n=[q]+1,
$$

where $[q]$ denotes the integer part of the real number $q$.

The following lemma obtained in [1] is useful in the rest of the paper.

Lemma 2.1 ([1]) For a given $y \in C([0, T], \mathbb{R})$, the unique solution of the fractional boundary value problem

$$
\left\{\begin{array}{l}
{ }^{c} D^{\alpha} x(t)=y(t), \quad t \in[0, T], 1<\alpha \leq 2, \\
a_{1} x(0)+b_{1}\left({ }^{c} D^{\gamma} x(0)\right)=c_{1}, \quad a_{2} x(T)+b_{2}\left({ }^{c} D^{\gamma} x(T)\right)=c_{2}, \quad 0<\gamma<1,
\end{array}\right.
$$

is given by

$$
\begin{aligned}
x(t)= & \int_{0}^{t} \frac{(t-s)^{\alpha-1}}{\Gamma(\alpha)} y(s) d s-\frac{t}{v_{1}}\left\{a_{2} \int_{0}^{T} \frac{(T-s)^{\alpha-1}}{\Gamma(\alpha)} y(s) d s\right. \\
& \left.+b_{2} \int_{0}^{T} \frac{(T-s)^{\alpha-\gamma-1}}{\Gamma(\alpha-\gamma)} y(s) d s\right\}+v_{2} t+\frac{c_{1}}{a_{1}}
\end{aligned}
$$

where

$$
v_{1}=\frac{a_{2} T \Gamma(2-\gamma)+b_{2} T^{1-\gamma}}{\Gamma(2-\gamma)}, \quad v_{2}=\frac{a_{1} c_{2}-a_{2} c_{1}}{a_{1} v_{1}} .
$$


We notice that the solution (4) of the problem (3) does not depend on the parameter $b_{1}$, that is to say, the parameter $b_{1}$ is of arbitrary nature for this problem. And by (4), we should assume that $a_{2} T^{\gamma} \Gamma(2-\gamma) \neq-b_{2}$.

Let $C([0, T], \mathbb{R})$ be the space of all continuous functions defined on $[0, T]$. Define the space $\mathcal{X}=\left\{x: x\right.$ and $\left.{ }^{c} D^{\beta} x \in C([0, T], \mathbb{R})\right\}(0<\beta \leq 1)$ endowed with the norm $\|x\|=$ $\max _{t \in[0, T]}|x(t)|+\left.\max _{t \in[0, T]}\right|^{c} D^{\beta} x(t) \mid$. We know that $(\mathcal{X},\|\cdot\|)$ is a Banach space.

Theorem 2.1 (Schauder fixed point theorem) Let $U$ be a closed, convex and nonempty subset of a Banach space $X$, let $P: U \rightarrow U$ be a continuous mapping such that $P(U)$ is a relatively compact subset of $X$. Then $P$ has at least one fixed point in $U$.

Theorem 2.2 (Nonlinear alternative for single-valued maps) Let $X$ be a Banach space, let $C$ be a closed, convex subset of $X$, let $U$ be an open subset of $C$ and $0 \in U$. Suppose that $P: \bar{U} \rightarrow C$ is a continuous and compact map. Then either (a) $P$ has a fixed point in $\bar{U}$, or (b) there exist an $x \in \partial U$ (the boundary of $U$ ) and $\lambda \in(0,1)$ with $x=\lambda P(x)$.

\section{Existence results}

In this section, we give some existence results for the problem (1).

In view of Lemma 2.1, we define an operator $\mathcal{F}: \mathcal{X} \rightarrow \mathcal{X}$ as

$$
\begin{aligned}
(\mathcal{F} x)(t)= & \int_{0}^{t} \frac{(t-s)^{\alpha-1}}{\Gamma(\alpha)} f\left(s, x(s),{ }^{c} D^{\beta} x(s)\right) d s \\
& -\frac{t}{v_{1}}\left\{a_{2} \int_{0}^{T} \frac{(T-s)^{\alpha-1}}{\Gamma(\alpha)} f\left(s, x(s),{ }^{c} D^{\beta} x(s)\right) d s\right. \\
& \left.+b_{2} \int_{0}^{T} \frac{(T-s)^{\alpha-\gamma-1}}{\Gamma(\alpha-\gamma)} f\left(s, x(s),{ }^{c} D^{\beta} x(s)\right) d s\right\}+v_{2} t+\frac{c_{1}}{a_{1}} .
\end{aligned}
$$

It is clear that the problem (1) has solutions if and only if the operator equation $\mathcal{F} x=x$ has fixed points. For any $x \in \mathcal{X}$, let

$$
(\mathcal{N} x)(t)=f\left(t, x(t),{ }^{c} D^{\beta} x(t)\right), \quad t \in[0, T] .
$$

Since the function $f$ is continuous and

$$
\left({ }^{c} D^{\beta} \mathcal{F} x\right)(t)=\left(I^{\alpha-\beta} \mathcal{N} x\right)(t)-\frac{k t^{1-\beta}}{\Gamma(2-\beta)},
$$

we know that the operator $\mathcal{F}$ maps $\mathcal{X}$ into $\mathcal{X}$. Here $k$ is a constant given by

$$
k=\frac{1}{v_{1}}\left\{a_{2} \int_{0}^{T} \frac{(T-s)^{\alpha-1}}{\Gamma(\alpha)}(\mathcal{N} x)(s) d s+b_{2} \int_{0}^{T} \frac{(T-s)^{\alpha-\gamma-1}}{\Gamma(\alpha-\gamma)}(\mathcal{N} x)(s) d s\right\}-v_{2} .
$$

We put $\mathcal{F} x=\mathcal{F}_{1} x+\mathcal{F}_{2} x$, where

$$
\left(\mathcal{F}_{1} x\right)(t)=\int_{0}^{t} \frac{(t-s)^{\alpha-1}}{\Gamma(\alpha)}(\mathcal{N} x)(s) d s, \quad\left(\mathcal{F}_{2} x\right)(t)=-k_{x} t+\frac{c_{1}}{a_{1}} .
$$

Here $k_{x}$ means that the constant $k$ is related to $x$. 
Now we are in a position to present our main results. The methods used to prove the existence results are standard; however, their exposition in the framework of the problem (1) is new.

Theorem 3.1 Suppose that the continuous function $f$ satisfies the following assumption:

$$
\begin{aligned}
& \left|f\left(t, x_{1}, y_{1}\right)-f\left(t, x_{2}, y_{2}\right)\right| \leq m(t)\left(\left|x_{1}-x_{2}\right|+\left|y_{1}-y_{2}\right|\right) \\
& \text { for } t \in[0, T], x_{i}, y_{i} \in \mathbb{R}, i=1,2 \text { and } m \in L^{\frac{1}{\tau}}\left([0, T], \mathbb{R}^{+}\right), \tau \in(0, \alpha-1) . \text { If } \\
& \frac{\|m\| T^{\alpha-\tau}}{\Gamma(\alpha)}\left(\frac{1-\tau}{\alpha-\tau}\right)^{1-\tau}\left(1+\frac{\left|a_{2}\right| T}{\left|v_{1}\right|}+\frac{\left|a_{2}\right| T^{1-\beta}}{\left|v_{1}\right| \Gamma(2-\beta)}\right) \\
& +\left(1+\frac{T^{-\beta}}{\Gamma(2-\beta)}\right) \frac{\|m\|\left|b_{2}\right| T^{\alpha-\gamma-\tau+1}}{\left|v_{1}\right| \Gamma(\alpha-\gamma)}\left(\frac{1-\tau}{\alpha-\gamma-\tau}\right)^{1-\tau} \\
& +\frac{\|m\| T^{\alpha-\beta-\tau}}{\Gamma(\alpha-\beta)}\left(\frac{1-\tau}{\alpha-\beta-\tau}\right)^{1-\tau}<1
\end{aligned}
$$

then the problem (1) has a unique solution.

Proof Denote $\|m\|=\left(\int_{0}^{T}|m(s)|^{\frac{1}{\tau}} d s\right)^{\tau}$. For any $x, y \in \mathcal{X}$ and for each $t \in[0, T]$, by the Hölder inequality, we have

$$
\begin{aligned}
\left|\left(\mathcal{F}_{1} x\right)(t)-\left(\mathcal{F}_{1} y\right)(t)\right| & \\
= & \left|\int_{0}^{t} \frac{(t-s)^{\alpha-1}}{\Gamma(\alpha)}((\mathcal{N} x)(s)-(\mathcal{N} y)(s)) d s\right| \\
\leq & \left|\int_{0}^{t} \frac{(t-s)^{\alpha-1}}{\Gamma(\alpha)} m(s)\left(|x(s)-y(s)|+\left|{ }^{c} D^{\beta} x(s)-{ }^{c} D^{\beta} y(s)\right|\right) d s\right| \\
\leq & \frac{\|m\|\|x-y\|}{\Gamma(\alpha)}\left(\frac{1-\tau}{\alpha-\tau}\right)^{1-\tau} T^{\alpha-\tau}, \\
\left|\left(\mathcal{F}_{2} x\right)(t)-\left(\mathcal{F}_{2} y\right)(t)\right| & \\
= & \left|t\left(k_{x}-k_{y}\right)\right| \\
\leq & T \mid \frac{a_{2}}{v_{1}} \int_{0}^{T} \frac{(T-s)^{\alpha-1}}{\Gamma(\alpha)}((\mathcal{N} x)(s)-(\mathcal{N} y)(s)) d s \\
& +\frac{b_{2}}{v_{1}} \int_{0}^{T} \frac{(T-s)^{\alpha-\gamma-1}}{\Gamma(\alpha-\gamma)}((\mathcal{N} x)(s)-(\mathcal{N} y)(s)) d s \mid \\
\leq & \frac{\|m\|}{\left|v_{1}\right|}\left\{\frac{\left|a_{2}\right| T^{\alpha-\tau+1}}{\Gamma(\alpha)}\left(\frac{1-\tau}{\alpha-\tau}\right)^{1-\tau}+\frac{\left|b_{2}\right| T^{\alpha-\gamma-\tau+1}}{\Gamma(\alpha-\gamma)}\left(\frac{1-\tau}{\alpha-\gamma-\tau}\right)^{1-\tau}\right\}\|x-y\| .
\end{aligned}
$$

Similarly, we have

$$
\begin{aligned}
& \left|\left({ }^{c} D^{\beta} \mathcal{F} x\right)(t)-\left({ }^{c} D^{\beta} \mathcal{F} y\right)(t)\right| \\
& \quad=\left|\left(I^{\alpha-\beta} \mathcal{N} x\right)(t)-\frac{k_{x} t^{1-\beta}}{\Gamma(2-\beta)}-\left(I^{\alpha-\beta} \mathcal{N} y\right)(t)+\frac{k_{y} t^{1-\beta}}{\Gamma(2-\beta)}\right|
\end{aligned}
$$




$$
\begin{aligned}
\leq & \frac{\|m\| T^{\alpha-\beta-\tau}}{\Gamma(\alpha-\beta)}\left(\frac{1-\tau}{\alpha-\beta-\tau}\right)^{1-\tau}\|x-y\|+\frac{\|m\| T^{1-\beta}}{\left|v_{1}\right| \Gamma(2-\beta)} \\
& \times\left\{\frac{\left|a_{2}\right| T^{\alpha-\tau}}{\Gamma(\alpha)}\left(\frac{1-\tau}{\alpha-\tau}\right)^{1-\tau}+\frac{\left|b_{2}\right| T^{\alpha-\gamma-\tau}}{\Gamma(\alpha-\gamma)}\left(\frac{1-\tau}{\alpha-\gamma-\tau}\right)^{1-\tau}\right\}\|x-y\| .
\end{aligned}
$$

From the above inequalities, we obtain

$$
\begin{aligned}
\|\mathcal{F} x-\mathcal{F} y\| \leq & \left\{\frac{\|m\| T^{\alpha-\tau}}{\Gamma(\alpha)}\left(\frac{1-\tau}{\alpha-\tau}\right)^{1-\tau}\left(1+\frac{\left|a_{2}\right| T}{\left|v_{1}\right|}+\frac{\left|a_{2}\right| T^{1-\beta}}{\left|\nu_{1}\right| \Gamma(2-\beta)}\right)\right. \\
& +\left(1+\frac{T^{-\beta}}{\Gamma(2-\beta)}\right) \frac{\|m\|\left|b_{2}\right| T^{\alpha-\gamma-\tau+1}}{\left|v_{1}\right| \Gamma(\alpha-\gamma)}\left(\frac{1-\tau}{\alpha-\gamma-\tau}\right)^{1-\tau} \\
& \left.+\frac{\|m\| T^{\alpha-\beta-\tau}}{\Gamma(\alpha-\beta)}\left(\frac{1-\tau}{\alpha-\beta-\tau}\right)^{1-\tau}\right\}\|x-y\| .
\end{aligned}
$$

It follows from (7) that $\mathcal{F}$ is a contraction mapping. Hence the Banach fixed point theorem implies that $\mathcal{F}$ has a unique fixed point which is the unique solution of the problem (1). This is the end of the proof.

Corollary 3.1 Suppose that the continuous function $f$ satisfies

$$
\left|f\left(t, x_{1}, y_{1}\right)-f\left(t, x_{2}, y_{2}\right)\right| \leq H\left(\left|x_{1}-x_{2}\right|+\left|y_{1}-y_{2}\right|\right)
$$

for $t \in[0, T], x_{i}, y_{i} \in \mathbb{R}, i=1,2$, and $H>0$ is a constant. If

$$
\begin{aligned}
& \frac{H T^{\alpha}}{\Gamma(\alpha+1)}\left(1+\frac{\left|a_{2}\right| T}{\left|v_{1}\right|}+\frac{\left|a_{2}\right| T^{1-\beta}}{\left|v_{1}\right| \Gamma(2-\beta)}\right)+\frac{H T^{\alpha-\beta}}{\Gamma(\alpha-\beta+1)} \\
& \quad+\left(1+\frac{T^{-\beta}}{\Gamma(2-\beta)}\right) \frac{H\left|b_{2}\right| T^{\alpha-\gamma+1}}{\left|v_{1}\right| \Gamma(\alpha-\gamma+1)}<1,
\end{aligned}
$$

then the problem (1) has a unique solution.

Theorem 3.2 Suppose that there exist a constant $\tau \in(0, \alpha-1)$ and a function $m \in$ $L^{\frac{1}{\tau}}\left([0, T], \mathbb{R}^{+}\right)$such that

$$
|f(t, x, y)| \leq m(t)+d_{1}|x|^{\rho_{1}}+d_{2}|y|^{\rho_{2}}
$$

where $d_{i} \geq 0,0 \leq \rho_{i}<1$ for $i=1,2$. Then the problem (1) has at least one solution.

Proof Denote $\|m\|=\left(\int_{0}^{T}|m(s)|^{\frac{1}{\tau}} d s\right)^{\tau}$. Let $B_{r}=\{x \in \mathcal{X}:\|x\| \leq r\}$, and $r>0$ is a positive number which will be given below (see (9)). It is clear that $B_{r}$ is a closed, bounded and convex subset of the Banach space $\mathcal{X}$.

The operator $\mathcal{F}$ maps $B_{r}$ into $B_{r}$. For any $x \in B_{r}$, we have

$$
\begin{aligned}
\left|\left(\mathcal{F}_{1} x\right)(t)\right| & \leq \frac{1}{\Gamma(\alpha)} \int_{0}^{t}(t-s)^{\alpha-1} m(s) d s+\frac{d_{1} r^{\rho_{1}}+d_{2} r^{\rho_{2}}}{\Gamma(\alpha)} \int_{0}^{t}(t-s)^{\alpha-1} d s \\
& \leq \frac{\|m\| T^{\alpha-\tau}}{\Gamma(\alpha)}\left(\frac{1-\tau}{\alpha-\tau}\right)^{1-\tau}+\frac{\left(d_{1} r^{\rho_{1}}+d_{2} r^{\rho_{2}}\right) T^{\alpha}}{\Gamma(\alpha+1)}
\end{aligned}
$$




$$
\begin{aligned}
&\left|\left(\mathcal{F}_{2} x\right)(t)\right| \leq\left|k_{x}\right| T+\frac{\left|c_{1}\right|}{\left|a_{1}\right|} \\
&\left|k_{x}\right| \leq\left|v_{2}\right|+\frac{1}{\left|v_{1}\right|}\left\{\frac{\left|a_{2}\right|\|m\| T^{\alpha-\tau}}{\Gamma(\alpha)}\left(\frac{1-\tau}{\alpha-\tau}\right)^{1-\tau}+\frac{\left|a_{2}\right|\left(d_{1} r^{\rho_{1}}+d_{2} r^{\rho_{2}}\right) T^{\alpha}}{\Gamma(\alpha+1)}\right. \\
&\left.\quad+\frac{\left|b_{2}\right|\|m\| T^{\alpha-\gamma-\tau}}{\Gamma(\alpha-\gamma)}\left(\frac{1-\tau}{\alpha-\gamma-\tau}\right)^{1-\tau}+\frac{\left|b_{2}\right|\left(d_{1} r^{\rho_{1}}+d_{2} r^{\rho_{2}}\right) T^{\alpha-\gamma}}{\Gamma(\alpha-\gamma+1)}\right\} .
\end{aligned}
$$

So, we have

$$
\begin{aligned}
|(\mathcal{F} x)(t)| \leq & \frac{\left|c_{1}\right|}{\left|a_{1}\right|}+\left|v_{2}\right| T+\frac{\|m\| T^{\alpha-\tau}}{\Gamma(\alpha)}\left(\frac{1-\tau}{\alpha-\tau}\right)^{1-\tau}\left(1+\frac{\left|a_{2}\right| T}{\left|v_{1}\right|}\right) \\
& +\frac{\left|b_{2}\right|\|m\| T^{\alpha-\gamma-\tau+1}}{\left|\nu_{1}\right| \Gamma(\alpha-\gamma)}\left(\frac{1-\tau}{\alpha-\gamma-\tau}\right)^{1-\tau}+\left(d_{1} r^{\rho_{1}}+d_{2} r^{\rho_{2}}\right) \\
& \times\left(\frac{T^{\alpha}}{\Gamma(\alpha+1)}+\frac{\left|a_{2}\right| T^{\alpha+1}}{\left|\nu_{1}\right| \Gamma(\alpha+1)}+\frac{\left|b_{2}\right| T^{\alpha-\gamma+1}}{\left|\nu_{1}\right| \Gamma(\alpha-\gamma+1)}\right) .
\end{aligned}
$$

Since

$$
\begin{aligned}
\left|\left(I^{\alpha-\beta} \mathcal{N} x\right)(t)\right| \leq & \frac{1}{\Gamma(\alpha-\beta)} \int_{0}^{t}(t-s)^{\alpha-\beta-1} m(s) d s \\
& +\frac{d_{1} r^{\rho_{1}}+d_{2} r^{\rho_{2}}}{\Gamma(\alpha-\beta)} \int_{0}^{t}(t-s)^{\alpha-\beta-1} d s \\
\leq & \frac{\|m\| T^{\alpha-\beta-\tau}}{\Gamma(\alpha-\beta)}\left(\frac{1-\tau}{\alpha-\beta-\tau}\right)^{1-\tau}+\frac{\left(d_{1} r^{\rho_{1}}+d_{2} r^{\rho_{2}}\right) T^{\alpha-\beta}}{\Gamma(\alpha-\beta+1)},
\end{aligned}
$$

then from (6) and the estimation of $k_{x}$, we have

$$
\begin{aligned}
& \left|\left({ }^{c} D^{\beta} \mathcal{F} x\right)(t)\right| \\
& \leq \leq \\
& \quad \frac{\|m\| T^{\alpha-\beta-\tau}}{\Gamma(\alpha-\beta)}\left(\frac{1-\tau}{\alpha-\beta-\tau}\right)^{1-\tau}+\frac{T^{1-\beta}\left|\nu_{2}\right|}{\Gamma(2-\beta)} \\
& \quad+\frac{\|m\| T^{1-\beta}}{\left|v_{1}\right| \Gamma(2-\beta)}\left(\frac{\left|a_{2}\right| T^{\alpha-\tau}}{\Gamma(\alpha)}\left(\frac{1-\tau}{\alpha-\tau}\right)^{1-\tau}+\frac{\left|b_{2}\right| T^{\alpha-\gamma-\tau}}{\Gamma(\alpha-\gamma)}\left(\frac{1-\tau}{\alpha-\gamma-\tau}\right)^{1-\tau}\right) \\
& \quad+\left(d_{1} r^{\rho_{1}}+d_{2} r^{\rho_{2}}\right)\left\{\frac{T^{\alpha-\beta}}{\Gamma(\alpha-\beta+1)}+\frac{T^{1-\beta}}{\left|v_{1}\right| \Gamma(2-\beta)}\left(\frac{\left|a_{2}\right| T^{\alpha}}{\Gamma(\alpha+1)}+\frac{\left|b_{2}\right| T^{\alpha-\gamma}}{\Gamma(\alpha-\gamma+1)}\right)\right\} .
\end{aligned}
$$

Denote

$$
\begin{aligned}
L= & \frac{\left|c_{1}\right|}{\left|a_{1}\right|}+\left|v_{2}\right| T+\frac{\|m\| T^{\alpha-\tau}}{\Gamma(\alpha)}\left(\frac{1-\tau}{\alpha-\tau}\right)^{1-\tau}\left(1+\frac{\left|a_{2}\right| T}{\left|v_{1}\right|}\right)+\frac{T^{1-\beta}\left|v_{2}\right|}{\Gamma(2-\beta)} \\
& +\frac{\left|b_{2}\right|\|m\| T^{\alpha-\gamma-\tau+1}}{\left|v_{1}\right| \Gamma(\alpha-\gamma)}\left(\frac{1-\tau}{\alpha-\gamma-\tau}\right)^{1-\tau}+\frac{\|m\| T^{\alpha-\beta-\tau}}{\Gamma(\alpha-\beta)}\left(\frac{1-\tau}{\alpha-\beta-\tau}\right)^{1-\tau} \\
& +\frac{\|m\| T^{1-\beta}}{\left|v_{1}\right| \Gamma(2-\beta)}\left(\frac{\left|a_{2}\right| T^{\alpha-\tau}}{\Gamma(\alpha)}\left(\frac{1-\tau}{\alpha-\tau}\right)^{1-\tau}+\frac{\left|b_{2}\right| T^{\alpha-\gamma-\tau}}{\Gamma(\alpha-\gamma)}\left(\frac{1-\tau}{\alpha-\gamma-\tau}\right)^{1-\tau}\right), \\
M= & \frac{T^{\alpha}}{\Gamma(\alpha+1)}+\frac{\left|a_{2}\right| T^{\alpha+1}}{\left|v_{1}\right| \Gamma(\alpha+1)}+\frac{\left|b_{2}\right| T^{\alpha-\gamma+1}}{\left|v_{1}\right| \Gamma(\alpha-\gamma+1)} \\
& +\frac{T^{\alpha-\beta}}{\Gamma(\alpha-\beta+1)}+\frac{T^{1-\beta}}{\left|v_{1}\right| \Gamma(2-\beta)}\left(\frac{\left|a_{2}\right| T^{\alpha}}{\Gamma(\alpha+1)}+\frac{\left|b_{2}\right| T^{\alpha-\gamma}}{\Gamma(\alpha-\gamma+1)}\right) .
\end{aligned}
$$


Now let $r$ be a positive number such that

$$
r \geq \max \left\{3 L,\left(3 M d_{1}\right)^{\frac{1}{1-\rho_{1}}},\left(3 M d_{2}\right)^{\frac{1}{1-\rho_{2}}}\right\} .
$$

Then it is obvious that for any $x \in B_{r}$,

$$
\|\mathcal{F} x\| \leq L+M\left(d_{1} r^{\rho_{1}}+d_{2} r^{\rho_{2}}\right) \leq \frac{r}{3}+\frac{r}{3}+\frac{r}{3}=r .
$$

It is easy to verify that the operator $\mathcal{F}$ is continuous since $f$ is continuous. Next, we show that $\mathcal{F}$ is equicontinuous on bounded subsets of $\mathcal{X}$. Let $\bar{B}$ be any bounded subset of $\mathcal{X}$. Since $f$ is continuous, we can assume, without any loss of generality, that $\left|f\left(t, x(t),{ }^{c} D^{\beta} x(t)\right)\right| \leq N$ for any $x \in \bar{B}$ and $t \in[0, T]$.

Now let $0 \leq t_{1}<t_{2} \leq T$. We have the following facts:

$$
\begin{aligned}
& \left|\left(\mathcal{F}_{1} x\right)\left(t_{2}\right)-\left(\mathcal{F}_{1} x\right)\left(t_{1}\right)\right| \\
& =\left|\int_{t_{1}}^{t_{2}} \frac{\left(t_{2}-s\right)^{\alpha-1}}{\Gamma(\alpha)}(\mathcal{N} x)(s) d s+\int_{0}^{t_{1}} \frac{\left(t_{2}-s\right)^{\alpha-1}-\left(t_{1}-s\right)^{\alpha-1}}{\Gamma(\alpha)}(\mathcal{N} x)(s) d s\right| \\
& \leq \frac{N\left(t_{2}-t_{1}\right)^{\alpha}}{\Gamma(\alpha+1)}+\frac{N\left|t_{2}^{\alpha}-\left(t_{2}-t_{1}\right)^{\alpha}-t_{1}^{\alpha}\right|}{\Gamma(\alpha+1)} \\
& \quad \leq \frac{2 N\left(t_{2}-t_{1}\right)^{\alpha}}{\Gamma(\alpha+1)}+\frac{N\left|t_{2}^{\alpha}-t_{1}^{\alpha}\right|}{\Gamma(\alpha+1)} \\
& \left|\left(\mathcal{F}_{2} x\right)\left(t_{2}\right)-\left(\mathcal{F}_{2} x\right)\left(t_{1}\right)\right|=\left|-k_{x} t_{2}+\frac{c_{1}}{a_{1}}+k_{x} t_{1}-\frac{c_{1}}{a_{1}}\right| \\
& \quad \leq\left(\frac{N}{\left|v_{1}\right|}\left(\frac{\left|a_{2}\right| T^{\alpha}}{\Gamma(\alpha+1)}+\frac{\left|b_{2}\right| T^{\alpha-\gamma}}{\Gamma(\alpha-\gamma+1)}\right)+\left|v_{2}\right|\right)\left(t_{2}-t_{1}\right), \\
& =\left|\left({ }^{c} D^{\beta} \mathcal{F} x\right)\left(t_{2}\right)-\left({ }^{c} D^{\beta} \mathcal{F} x\right)\left(t_{1}\right)\right| \\
& \quad \leq \frac{1}{\Gamma(2-\beta)}\left(\frac{N}{\left|v_{1}\right|}\left(\frac{\left.\left|t_{2}\right| T^{\alpha}\right)-}{\Gamma(\alpha+1)}+\frac{\left|b_{2}\right| T^{\alpha-\gamma}}{\Gamma(\alpha-\gamma+1)}\right)+\left|v_{2}\right|\right)\left|t_{2}^{1-\beta}-t_{1}^{1-\beta}\right| \\
& \quad+\frac{N\left|t_{2}^{\alpha-\beta}-t_{1}^{\alpha-\beta}\right|}{\Gamma(\alpha-\beta+1)}+\frac{2 N\left(t_{2}-t_{1}\right)^{\alpha-\beta}}{\Gamma(\alpha-\beta+1)} .
\end{aligned}
$$

Hence we have (since $\alpha>1, \alpha-\beta>0$ and $1-\beta \geq 0$ )

$$
\left\|(\mathcal{F} x)\left(t_{2}\right)-(\mathcal{F} x)\left(t_{1}\right)\right\| \rightarrow 0 \quad \text { as } t_{2} \rightarrow t_{1}
$$

and the limit is independent of $x \in \bar{B}$. Therefore the operator $\mathcal{F}: B_{r} \rightarrow B_{r}$ is equicontinuous and uniformly bounded. The Arzela-Ascoli theorem implies that $\mathcal{F}\left(B_{r}\right)$ is relatively compact in $\mathcal{X}$.

From Theorem 2.1, the problem (1) has at least one solution. The proof is completed.

Corollary 3.2 Assume that $|f(t, x, y)| \leq v(t)$ for $t \in[0, T], x, y \in \mathbb{R}$ with $v \in C\left([0, T], \mathbb{R}^{+}\right)$. Then the problem (1) has at least one solution. 
In this situation, since for any $\tau \in(0, \alpha-1), v \in L^{\frac{1}{\tau}}\left([0, T], \mathbb{R}^{+}\right)$, then let $d_{1}=d_{2}=0$ in Theorem 3.2, we get the result.

Corollary 3.3 Assume that there exist a constant $\tau \in(0, \alpha-1)$ and a function $m \in$ $L^{\frac{1}{\tau}}\left([0, T], \mathbb{R}^{+}\right)$such that

$$
|f(t, x, y)| \leq m(t)+d_{1}|x|+d_{2}|y|, \quad d_{i} \geq 0, i=1,2 .
$$

If $\left(d_{1}+d_{2}\right) M<1(M$ is defined by (3)), then the problem (1) has at least one solution.

The proof of this corollary is similar to Theorem 3.2.

Theorem 3.3 Assume that: (1) there exist two nondecreasing functions $\rho_{1}, \rho_{2}:[0, \infty) \rightarrow$ $[0, \infty)$ and a function $m \in L^{\frac{1}{\tau}}\left([0, T], \mathbb{R}^{+}\right)$with $\tau \in(0, \alpha-1)$ such that

$$
|f(t, x, y)| \leq m(t)\left(\rho_{1}(|x|)+\rho_{2}(|y|)\right)
$$

for $t \in[0, T]$ and $x, y \in \mathbb{R}$.

(2) There exists a constant $Z>0$ such that

$$
\frac{Z}{T\left|\nu_{2}\right|+\frac{\left|c_{1}\right|}{\left|a_{1}\right|}+\frac{T^{1-\beta}}{\Gamma(2-\beta)}\left|\nu_{2}\right|+\left(\rho_{1}(Z)+\rho_{2}(Z)\right)\|m\| \Delta}>1
$$

here $\|m\|=\left(\int_{0}^{T}|m(s)|^{\frac{1}{\tau}} d s\right)^{\tau}$ and $\Delta$ denotes the following number:

$$
\begin{aligned}
& \left\{\frac{T^{\alpha-\tau}}{\Gamma(\alpha)}\left(\frac{1-\tau}{\alpha-\tau}\right)^{1-\tau}+\frac{T^{\alpha-\beta-\tau}}{\Gamma(\alpha-\beta)}\left(\frac{1-\tau}{\alpha-\beta-\tau}\right)^{1-\tau}+\left(\frac{T}{\left|\nu_{1}\right|}+\frac{T^{1-\beta}}{\left|\nu_{1}\right| \Gamma(2-\beta)}\right)\right. \\
& \left.\quad \times\left(\frac{\left|a_{2}\right| T^{\alpha-\tau}}{\Gamma(\alpha)}\left(\frac{1-\tau}{\alpha-\tau}\right)^{1-\tau}+\frac{\left|b_{2}\right| T^{\alpha-\gamma-\tau}}{\Gamma(\alpha-\gamma)}\left(\frac{1-\tau}{\alpha-\gamma-\tau}\right)^{1-\tau}\right)\right\} .
\end{aligned}
$$

Then the problem (1) has at least one solution.

Proof Firstly, we show that the operator $\mathcal{F}$ defined by (5) maps bounded sets into bounded sets in the space $\mathcal{X}$. Let $B_{r}=\{x: x \in \mathcal{X}$ and $\|x\| \leq r\}, r>0$. For any $x \in B_{r}$, we have

$$
\begin{aligned}
\left|\left(\mathcal{F}_{1} x\right)(t)\right|= & \left|\frac{1}{\Gamma(\alpha)} \int_{0}^{t}(t-s)^{\alpha-1}(\mathcal{N} x)(s) d s\right| \\
\leq & \frac{\rho_{1}(r)+\rho_{2}(r)}{\Gamma(\alpha)} \int_{0}^{t}(t-s)^{\alpha-1} m(s) d s \\
\leq & \frac{\left(\rho_{1}(r)+\rho_{2}(r)\right)\|m\| T^{\alpha-\tau}}{\Gamma(\alpha)}\left(\frac{1-\tau}{\alpha-\tau}\right)^{1-\tau}, \\
\left|\left(\mathcal{F}_{2} x\right)(t)\right| \leq & T\left|\nu_{2}\right|+\frac{T\|m\|\left(\rho_{1}(r)+\rho_{2}(r)\right)}{\left|v_{1}\right|}\left\{\frac{\left|a_{2}\right| T^{\alpha-\tau}}{\Gamma(\alpha)}\left(\frac{1-\tau}{\alpha-\tau}\right)^{1-\tau}\right. \\
& \left.+\frac{\left|b_{2}\right| T^{\alpha-\gamma-\tau}}{\Gamma(\alpha-\gamma)}\left(\frac{1-\tau}{\alpha-\gamma-\tau}\right)^{1-\tau}\right\}+\frac{\left|c_{1}\right|}{\left|a_{1}\right|},
\end{aligned}
$$




$$
\begin{aligned}
\left|\left({ }^{c} D^{\beta} \mathcal{F} x\right)(t)\right| \leq & \left|\left(I^{\alpha-\beta} \mathcal{N} x\right)(t)\right|+\frac{T^{1-\beta}}{\Gamma(2-\beta)}\left|k_{x}\right| \\
\leq & \frac{\left(\rho_{1}(r)+\rho_{2}(r)\right)\|m\| T^{\alpha-\beta-\tau}}{\Gamma(\alpha-\beta)}\left(\frac{1-\tau}{\alpha-\beta-\tau}\right)^{1-\tau} \\
& +\frac{T^{1-\beta}}{\Gamma(2-\beta)}\left\{\left|v_{2}\right|+\frac{\|m\|\left(\rho_{1}(r)+\rho_{2}(r)\right)}{\left|\nu_{1}\right|}\right. \\
& \left.\times\left(\frac{\left|a_{2}\right| T^{\alpha-\tau}}{\Gamma(\alpha)}\left(\frac{1-\tau}{\alpha-\tau}\right)^{1-\tau}+\frac{\left|b_{2}\right| T^{\alpha-\gamma-\tau}}{\Gamma(\alpha-\gamma)}\left(\frac{1-\tau}{\alpha-\gamma-\tau}\right)^{1-\tau}\right)\right\} .
\end{aligned}
$$

Therefore we have

$$
\begin{aligned}
\|\mathcal{F} x\| \leq & T\left|v_{2}\right|+\frac{\left|c_{1}\right|}{\left|a_{1}\right|}+\frac{T^{1-\beta}}{\Gamma(2-\beta)}\left|v_{2}\right|+\left(\rho_{1}(r)+\rho_{2}(r)\right)\|m\| \\
& \times\left\{\frac{T^{\alpha-\tau}}{\Gamma(\alpha)}\left(\frac{1-\tau}{\alpha-\tau}\right)^{1-\tau}+\frac{T^{\alpha-\beta-\tau}}{\Gamma(\alpha-\beta)}\left(\frac{1-\tau}{\alpha-\beta-\tau}\right)^{1-\tau}+\left(\frac{T}{\left|v_{1}\right|}+\frac{T^{1-\beta}}{\left|\nu_{1}\right| \Gamma(2-\beta)}\right)\right. \\
& \left.\times\left(\frac{\left|a_{2}\right| T^{\alpha-\tau}}{\Gamma(\alpha)}\left(\frac{1-\tau}{\alpha-\tau}\right)^{1-\tau}+\frac{\left|b_{2}\right| T^{\alpha-\gamma-\tau}}{\Gamma(\alpha-\gamma)}\left(\frac{1-\tau}{\alpha-\gamma-\tau}\right)^{1-\tau}\right)\right\}
\end{aligned}
$$

That is to say, we have

$$
\|\mathcal{F} x\| \leq T\left|v_{2}\right|+\frac{\left|c_{1}\right|}{\left|a_{1}\right|}+\frac{T^{1-\beta}}{\Gamma(2-\beta)}\left|v_{2}\right|+\left(\rho_{1}(r)+\rho_{2}(r)\right)\|m\| \Delta .
$$

Secondly, we claim that $\mathcal{F}$ is equicontinuous on bounded sets of $\mathcal{X}$. To prove it, we only need to repeat verbatim the corresponding part in the proof of Theorem 3.2.

Finally, for $\lambda \in(0,1)$, let $x=\lambda \mathcal{F} x$. Due to (11), we have

$$
\|x\|=\|\lambda \mathcal{F} x\| \leq T\left|v_{2}\right|+\frac{\left|c_{1}\right|}{\left|a_{1}\right|}+\frac{T^{1-\beta}}{\Gamma(2-\beta)}\left|\nu_{2}\right|+\left(\rho_{1}(\|x\|)+\rho_{2}(\|x\|)\right)\|m\| \Delta .
$$

On the other hand, we have

$$
\frac{\|x\|}{T\left|\nu_{2}\right|+\frac{\left|c_{1}\right|}{\left|a_{1}\right|}+\frac{T^{1-\beta}}{\Gamma(2-\beta)}\left|\nu_{2}\right|+\left(\rho_{1}(\|x\|)+\rho_{2}(\|x\|)\right)\|m\| \Delta} \leq 1 .
$$

From (10), there exists $Z>0$ such that $\|x\| \neq Z$. Define a set

$$
\mathcal{U}=\{x \in \mathcal{X}:\|x\|<Z\}
$$

It is obvious that the operator $\mathcal{F}: \overline{\mathcal{U}} \rightarrow \mathcal{X}$ is continuous and completely continuous. By the definition of the set $\mathcal{U}$, there is no $x \in \partial \mathcal{U}$ such that $x=\lambda \mathcal{F} x$ for some $0<\lambda<1$. Consequently, by Theorem 2.2, we obtain that $\mathcal{F}$ has a fixed point $x \in \overline{\mathcal{U}}$ which is a solution of the problem (1). This is the end of the proof.

\section{Examples}

Example 1 Let $T=1, \alpha=\frac{7}{4}, \beta=\frac{3}{4}$ and $\gamma=\frac{1}{4}$. We consider the boundary value problem

$$
\left\{\begin{array}{l}
{ }^{c} D^{\frac{7}{4}} x(t)=\frac{-3 \ln (t+1)}{2 \sin \left(t^{2}\right)+5}+\frac{1}{(t+3)^{2}}\left(\sin x(t)+\frac{\left|{ }^{c} D^{\frac{3}{4}} x(t)\right|}{1+{ }^{c} D^{\frac{3}{4}} x(t) \mid}\right), \quad t \in[0,1], \\
x(0)+b_{1}\left({ }^{c} D^{\frac{1}{4}} x(0)\right)=\frac{1}{2}, \quad \frac{1}{2} x(1)+\frac{1}{3}\left({ }^{c} D^{\frac{1}{4}} x(1)\right)=2 .
\end{array}\right.
$$


From (12), we know that

$$
f(t, x, y)=\frac{-3 \ln (t+1)}{2 \sin \left(t^{2}\right)+5}+\frac{1}{(t+3)^{2}}\left(\sin x+\frac{|y|}{1+|y|}\right)
$$

and $a_{1}=1, c_{1}=\frac{1}{2}, a_{2}=\frac{1}{2}, b_{2}=\frac{1}{3}$ and $c_{2}=2$. It is clear that

$$
\left|f\left(t, x_{1}, y_{1}\right)-f\left(t, x_{2}, y_{2}\right)\right| \leq \frac{1}{9}\left(\left|x_{1}-x_{2}\right|+\left|y_{1}-y_{2}\right|\right)
$$

and

$$
\begin{aligned}
& \frac{H T^{\alpha}}{\Gamma(\alpha+1)}\left(1+\frac{\left|a_{2}\right| T}{\left|v_{1}\right|}+\frac{\left|a_{2}\right| T^{1-\beta}}{\left|v_{1}\right| \Gamma(2-\beta)}\right)+\frac{H T^{\alpha-\beta}}{\Gamma(\alpha-\beta+1)} \\
& \quad+\left(1+\frac{T^{-\beta}}{\Gamma(2-\beta)}\right) \frac{H\left|b_{2}\right| T^{\alpha-\gamma+1}}{\left|v_{1}\right| \Gamma(\alpha-\gamma+1)} \approx \frac{1}{9}(1.2333+1+0.4933)<1 .
\end{aligned}
$$

Hence all the assumptions of Corollary 3.1 are satisfied. Therefore the problem (12) has a unique solution.

Example 2 Consider the following fractional differential equation:

$$
\left\{\begin{array}{l}
{ }^{c} D^{\frac{5}{3}} x(t)=\left(5 t^{2}-3 t\right) e^{-x^{2}(t)}+\frac{1}{2 \pi}|x(t)|^{\frac{1}{3}}+\left(\frac{\left.\right|^{c} D^{\frac{3}{4}} x(t) \mid}{1+\sin ^{2} x(t)}\right)^{\frac{1}{2}}, \quad t \in[0,1], \\
x(0)+b_{1}\left({ }^{c} D^{\frac{1}{2}} x(0)\right)=2.5, \quad 2 x(1)+\frac{1}{5}\left({ }^{c} D^{\frac{1}{2}} x(1)\right)=\pi .
\end{array}\right.
$$

In this case, we have

$$
f(t, x, y)=\left(5 t^{2}-3 t\right) e^{-x^{2}}+\frac{1}{2 \pi}|x|^{\frac{1}{3}}+\left(\frac{|y|}{1+\sin ^{2} x}\right)^{\frac{1}{2}}
$$

and $\alpha=\frac{5}{3}, \beta=\frac{3}{4}, \gamma=\frac{1}{2}, T=1, a_{1}=1, c_{1}=2.5, a_{2}=2, b_{2}=\frac{1}{5}, c_{2}=\pi$. Since

$$
|f(t, x, y)| \leq\left|5 t^{2}-3 t\right|+\frac{1}{2 \pi}|x|^{\frac{1}{3}}+|y|^{\frac{1}{2}}
$$

let $d_{1}=\frac{1}{2 \pi}, d_{2}=1, \rho_{1}=\frac{1}{3}, \rho_{2}=\frac{1}{2}$ and $m(t)=\left|5 t^{2}-3 t\right| \in L^{\infty}(0,1)$. Thus it follows from Theorem 3.2 that the problem (13) has at least one solution on $[0,1]$.

\section{Competing interests}

The authors declare that they have no competing interests.

\section{Authors' contributions}

The authors XL and ZL contributed to each part of this study equally and read and approved the final version of the manuscript.

\section{Acknowledgements}

Project is supported by NNSF of China (Grants No. 11271087, No. 61263006) and Guangxi Scientific Experimental (China-ASEAN Research) Centre No. 20120116. 


\section{References}

1. Ahmad, B, Ntouyas, SK: A note on fractional differential equations with fractional separated boundary conditions. Abstr. Appl. Anal. 2012, Article ID 818703 (2012)

2. Ahmad, B, Ntouyas, SK: Fractional differential inclusions with fractional separated boundary conditions. Fract. Calc. Appl. Anal. 15(3), 362-382 (2012)

3. Băleanu, D, Machado, JAT, Luo, ACJ: Fractional Dynamics and Control. Springer, Berlin (2012)

4. Sabatier, J, Agrawal, OP, Machado, JAT (eds.): Advances in Fractional Calculus: Theoretical Developments and Applications in Physics and Engineering. Springer, Dordrecht (2007)

5. Lakshmikantham, V, Leela, S, Vasundhara Devi, J: Theory of Fractional Dynamic Systems. Cambridge Scientific Publishers, Cambridge (2009)

6. Ahmad, B, Nieto, JJ: Existence results for a coupled system of nonlinear fractional differential equations with three-point boundary conditions. Comput. Math. Appl. 58, 1838-1843 (2009)

7. Ahmad, B, Ntouyas, SK: Fractional differential inclusions with fractional separated boundary conditions. Fract. Calc. Appl. Anal. 15(3), 362-382 (2012)

8. Agarwal, RP, Belmekki, M, Benchohra, M: A survey on semilinear differential equations and inclusions involving Riemann-Liouville fractional derivative. Adv. Differ. Equ. 2009, Article ID 981728 (2009)

9. Agarwal, RP, Benchohra, M, Hamani, S: A survey on existence results for boundary value problems of nonlinear fractional differential equations and inclusions. Acta Appl. Math. 109, 973-1033 (2010)

10. Bai, Z: On positive solutions of a nonlocal fractional boundary value problem. Nonlinear Anal. 72(2), 916-924 (2010)

11. Băleanu, D, Mustafa, OG, Agarwal, RP: An existence result for a superlinear fractional differential equation. Appl. Math. Lett. 23, 1129-1132 (2010)

12. Cernea, A: A note on the existence of solutions for some boundary value problems of fractional differential inclusions. Fract. Calc. Appl. Anal. 15(2), 183-194 (2012)

13. Chang, Y-K, Nieto, JJ: Some new existence results for fractional differential inclusions with boundary conditions. Math. Comput. Model. 49, 605-609 (2009)

14. Chen, A, Tian, Y: Existence of three positive solutions to three-point boundary value problem of nonlinear fractional differential equation. Differ. Equ. Dyn. Syst. 18(3), 327-339 (2010)

15. Lakshmikantham, V: Theory of fractional functional differential equations. Nonlinear Anal. 69, 3337-3343 (2008)

16. Liu, Z, Sun, J: Nonlinear boundary value problems of fractional differential systems. Comput. Math. Appl. 64(4), 463-475 (2012)

17. Li, CF, Luo, XN, Zhou, Y: Existence of positive solutions of the boundary value problem for nonlinear fractional differential equations. Comput. Math. Appl. 59, 1363-1375 (2010)

18. LV, LL, Wang, JR, Wei, W: Existence and uniqueness results for fractional differential equations with boundary value conditions. Opusc. Math. 31(4), 629-643 (2011)

19. Wang, G, Ahmad, B, Zhang, L: Impulsive anti-periodic boundary value problem for nonlinear differential equations of fractional order. Nonlinear Anal. 74(3), 792-804 (2011)

20. Wang, JR, Zhou, Y: Existence and controllability results for fractional semilinear differential inclusions. Nonlinear Anal., Real World Appl. 12(6), 3642-3653 (2011)

21. Wang, JR, LV, LL, Zhou, Y: Boundary value problems for fractional differential equations involving Caputo derivative in Banach spaces. J. Appl. Math. Comput. 38, 209-224 (2012)

22. Zhou, Y, Jiao, F: Nonlocal Cauchy problem for fractional evolution equations. Nonlinear Anal. 11, 4465-4475 (2010)

23. Ahmad, B, Nieto, JJ: Anti-periodic fractional boundary value problems with nonlinear term depending on lower order derivative. Fract. Calc. Appl. Anal. 15(3), 451-462 (2012)

24. Wang, F: Anti-periodic fractional boundary value problems for nonlinear differential equations of fractional order. Adv. Differ. Equ. 2012, 116 (2012)

25. Chen, A, Chen, Y: Existence of solutions to anti-periodic boundary value problem for nonlinear fractional differential equations. Differ. Equ. Dyn. Syst. 19(3), 237-252 (2011)

26. Kilbas, AA, Srivastava, HM, Trujillo, JJ: Theory and Applications of Fractional Differential Equations. North-Holland Mathematics Studies, vol. 204. Elsevier, Amsterdam (2006)

doi:10.1186/1687-1847-2013-78

Cite this article as: Liu and Liu: Separated boundary value problem for fractional differential equations depending on lower-order derivative. Advances in Difference Equations 2013 2013:78.

\section{Submit your manuscript to a SpringerOpen ${ }^{\odot}$ journal and benefit from:}

- Convenient online submission

- Rigorous peer review

- Immediate publication on acceptance

- Open access: articles freely available online

- High visibility within the field

- Retaining the copyright to your article 